2 Redefining human-animal relationships: an evaluation of methods to allow their empirical

3

4

5

6

7

8

9

$10 *$ Corresponding author

11 Dr Samantha J. Ward, Tel: +44 (0)115 8485239. Email: samantha.ward@ntu.ac.uk

\section{measurement in zoos}

Freisha Patel ${ }^{1}$, Katherine Whitehouse-Tedd ${ }^{1}$, Samantha J. Ward ${ }^{1 *}$

${ }^{1}$ School of Animal, Rural and Environmental Sciences, Nottingham Trent University, Southwell,

NG25 0QF, UK

12 
14 Scientific studies of human-animal interactions (HAIs) and how these develop into human-animal relationships (HARs) now represent some of the most significant contributions to animal welfare science. However, due to the current definition of HAR, studies have only been able to measure HAIs and infer its impact on HARs and animal welfare. Here we redefine HARs as a series of repeated HAIs between two individuals known to each other, the nature of which is influenced by their historical HAIs and considerations to the content, quality and the pattern of the interactions is also vital. With a new definition, it is now feasible to empirically measure HARs, however first it is important to evaluate current methods utilised in animal industries to allow standardisation across HAR research in zoos. Here we review the current methods that have been used to assess HAIs in animals, and determines their overall suitability for measuring HARs and their use in a zoo environment. Literature searches were conducted using the search terms "human-animal" AND "interaction", "human-animal" AND "relationship", "human-animal" AND bond". Subsequently, "zoo", "companion", "agriculture", "laboratory" and "wild" were added to each combination yielding five potential methods to evaluate. These methods were assessed according to a panel of indicators including reliability, robustness, practical application and feasibility for use in a zoo environment. Results indicated that the methods utilising 'latency', 'qualitative behaviour assessment' and the 'voluntary approach test' were potentially viable to assess HARs in a zoo environment, and could subsequently contribute to the assessment of welfare implications of these HARs for the animals involved. These methods now require empirical testing and comparisons within a zoo environment.

Keywords: Animal welfare, behaviour, human-animal-interactions, method, zoo

\subsection{Introduction}

The scientific study of human-animal interactions (HAI) is a multi-disciplinary field that is of interest to biologists, sociologists, psychologists, and conservationists, amongst others. An interaction has traditionally been defined in respect of inter-human interactions as a sequence in which an individual 
performs a behaviour towards another, which is subsequently responded to with a specific reaction (Hinde 1976, p3). According to Hinde (1976) a relationship is a succession of interactions between two individuals known to each other and influenced by a history of past interactions. Previous definitions of human-animal relationships (HARs) suggest that each participant within the relationship are able to make predictions about the other's responses (Estep \& Hetts 1992; Hemsworth et al 1993). However, if this definition is applied verbatim, it is not feasible to measure the ability of an animal to predict a human's behaviour. With this in mind, it appears necessary to devise a new definition that would enable the measurement of HARs. Therefore in this context and throughout this paper, a HAR is defined as a series of repeated HAIs between two individuals known to each other, the nature of which is influenced by their historical HAIs. Aspects to consider involve the content, quality and pattern of the interactions. For example, a HAR within a zoo setting can occur because of daily routine interactions with familiar zoo personnel (including keepers, education providers, maintenance and gardens teams, etc.). We propose that the HAR should be measured over a period of time, recording the content of interactions (i.e. the behaviours performed to create the interaction), the quality of interactions (i.e. positive, negative and/or neutral) and the order in which these interactions occur (i.e. the consistency of the interactions e.g. human A calls over animal B, B comes over to A, A strokes B or A calls over B, B slowly comes over to B with no further interactions). What is important to consider is that due to this definition, interactions between unfamiliar humans (e.g. visitors) cannot develop into HARs.

Within the agricultural animal sciences, methods to assess HAIs have been extensively studied and tested for validity and reliability. Similarly, zoo-based HAIs between animals and unfamiliar visitors have been relatively well investigated, albeit with varied outcomes. In contrast, empirical studies of HAIs with familiar humans in zoos (i.e. regular keepers), which have the potential to develop into HARs, have only recently begun to attract significant scientific attention (Hosey \& Melfi 2014). Moreover, the fundamental processes of method evaluation and standardisation are yet to be performed for HAI and HAR studies in zoos. This study addresses this knowledge gap by performing a systematic review of existing literature utilising methods designed to measure either HAI or HAR in zoo settings. 


\subsection{Human animal interactions and animal welfare}

Based on findings in companion (rabbits: Podberscek et al 1991), livestock (dairy calves: Ellingsen et al 2014), and laboratory (primates: Baker 2004) animals, HAIs and HARs represent a significant influencing factor in animal health and welfare, as well as having important roles to play in human health and wellbeing (O'Haire 2010). Likewise, HAIs and HARs have been recorded and investigated in zoos, and are considered to have implications for the health and welfare of the animals involved (HAR; Carlstead, 2009, Smith 2014, Martin \& Melfi 2016, Carlstead et al 2018, HAI; Carrasco et al 2009, Chelluri et al 2013, Ward \& Melfi 2013, 2015). However, most studies are primarily reliant on one method alone i.e. studies of animal behaviour and as welfare is multifaceted, additional measures would be needed to measure this specifically. According to our definition, the features of any interaction between an animal and a human will influence the way in which a HAR develops. Interactions can be perceived by the animal and the human as negative, neutral or positive, and consequently result in the development of a negative, neutral or positive HAR categorisation, respectively (Carlstead 2009, Hosey 2008; Smith 2014, Waiblinger et al 2006).

Previous research into the implications of HAIs for farm animal welfare have suggested that, whilst some animals may become accustomed to human contact, the majority of observed reactions involve some level of fear (Jones 1997). Many of the HAIs which occur in a farm environment are of a negative nature, such as exposure to rough handling, restraint and veterinary treatments, with positive or neutral HAIs generally only associated with feeding (Hemsworth \& Coleman 1998). It follows that assessments of HAIs and HARs based on the measurement of an animal's level of fear (or confidence) in humans have been well tested and are commonly used as part of on-farm welfare assessments with the use of both familiar and unfamiliar humans (Battini et al 2016; de Passillé \& Rushen 2005). However, fear induced responses from these negative HAIs can include attempts to escape, which in turn can result in injuries or deaths (i.e. from animals running into obstacles or other individuals) which raises welfare concerns and have detrimental health and safety implications in zoos. The use of an unfamiliar human within these tests primarily focusses on assessing an animal's general fear of humans, however based 
on the HAR definition in which a HAR can occur between two individuals known to each other, a familiar human would be used to explore this specific aspect.

The behaviour and attitude of stockpersons have shown to be major variables that can determine an animal's fear or confidence in a human, therefore stockmanship has the potential to influence the quality of HAIs and HARs as well as animal welfare. Positive or neutral HAIs and HARs can be beneficial to animal welfare, for example gentle handling or the presence of a familiar human may calm the animals in potentially negative situations, reducing the risk of injuries and therefore improving welfare (e.g. dairy cows; Waiblinger et al 2004). Ellingsen et al (2014) studied stockperson handling styles at 100 Norwegian dairy farms (100 stockpersons, mean number of 31 calves per farm) and identified differing styles which were termed 'positive interactions', 'calm/patient', 'dominating/aggressive', and 'insecure/nervous'. Results suggested that stockpersons who handled calves patiently and calmly were associated with animals exhibiting a higher level of positive mood, whereas those who used a nervous or dominating style of handling were associated with calves of a more negative mood. Positive moods in animals can be interpreted as pleasant emotions; this state is predicted to influence the nature of the HAI in a positive manner through a positive feedback cycle (see Waiblinger et al 2006). The emotional state of an animal during a HAI will likely influence its perception and reaction to humans and subsequently impact on the nature of the HAI itself; a range of influential factors contribute to this emotional dimension and must be considered during HAI studies, particularly when welfare is a measured outcome. In particular, fear and nervousness in animals has been associated with stress and reduced animal welfare (Rushen et al 1999). Waiblinger et al (2006) described the establishment of a negative feedback cycle between the handlers and animals, in which as the behaviour and attitude of stockperson worsens, fear subsequently increases for the animal, resulting in continued or increased negativity of the stockperson's attitude. For example, in Ellingsen's (2014) study, handling situations involving calves with a negative mood potentially led to animals which were more difficult to handle and uncooperative, resulting in more dominating or aggressive behaviour and attitude of handlers, and consequently a negative HAI and low welfare. In contrast, positive interactions that lead to pleasant emotions and a neutral or positive HAI, through the positive attitude of handlers, promotes good 
welfare. This is represented in one of the few zoo HAI studies relating to welfare; Ward and Melfi's (2013) zoo stockmanship cycle. This study highlighted the concept that a positive response from the animal following a positive keeper-animal interaction, promotes further positive responses from the animal and so forth, developing a positive HAR and potentially positive animal welfare. Positive HAIs have also been found to improve the welfare of laboratory animals; increased periods of positive HAIs have been shown to result in a reduction of fearful reactions in rabbits (Podberscek et al 1991), and to lower levels of abnormal behaviours in laboratory chimpanzees (Baker 2004).

Within companion animal HAR research, the typical focus is on how the relationship and/or an animalassisted therapy benefits human health and wellbeing (Walsh 2009), with only a few studies investigating the influence on animal welfare (Vitztum \& Urbanik 2016). However, factors including attachment level, anthropomorphism, and owner empathy and attitude towards their pets are elements which influence HAIs and consequently the animals' welfare (Ellingsen et al 2010; Marinelli et al 2007) or benefit to the human participant.

The importance of HAIs in animal welfare is an area of active research, and was highlighted as being one of the most significant recent contributions to zoo animal welfare science (Meehan et al 2016). Moreover, the connections among animal welfare, zoo visitor experience, and wildlife conservation are clear and notable in the revised vision of the World Association of Zoos and Aquaria (Barongi et al 2015). However, the role HAIs and HARs have in modulating an animals' behavioural repertoire, their social interactions, or life history events and outcomes has been inadequately explored, with only a few studies published to date (Mellen 1991, Wielebnowski 1999; Wielebnowski et al 2002, Carlstead 2009, Carrasco et al 2009, Smith 2014, Carlstead et al 2018). It is possible that the modulating effect of HAIs and HARs on these factors could exert significant influence on the welfare, management, and conservation consequences of zoo-housed animals. Whilst the extent of HAI influence has not yet been quantified in zoos, the potential exists for HAIs to impact on the welfare status of hundreds of thousands 
of animals, many of which are involved in captive breeding programmes of international significance to the $e x$-situ conservation of their species. For example, data retrieved on $31^{\text {st }}$ May 2018 from the 'Zoological Information Management System' (an animal records subscription-based database (Species360, 2018) revealed that there were 280,762 mammalia, 290,792 aves and 99,872 reptilia held in member facilities. These numbers are highly under-representative of zoos since it was estimated that within the $>10,000$ zoos worldwide (Fravel 2003), just over 10\% of these zoos contribute to this global database. The welfare implications of zoo HAIs and HARs therefore potentially affect a vast number of individual animals.

Despite this potential impact on animal welfare at the individual and population level, we have yet to establish a basic understanding of how HAIs and HARs function in zoos, or what the consequences of such HAIs and HARs may be for the diverse range of species in zoos. In addition, the results and discussion of published work on zoo HAR mainly refer to welfare as being related to HAIs or HARs rather than measuring welfare implications specifically. It is therefore important to firstly determine an appropriate method to measure HARs that can be applied in a standardised manner across a range of zoological settings and to a variety of species. Given the behavioural variation between species, let alone among different taxa, this is a difficult task. However, as the HAR is a product of HAIs, the response of the animal to a specific human stimuli can be measured as a fundamentally common starting point. As a first step towards this goal, this evaluation provides a comprehensive review of the current methods available to study HAIs and HARs from the perspective of the animal, the majority deriving from agricultural contexts (see Waiblinger et al 2006; Table 1). This will determine methods predicted to be suitable for testing in zoo environments, in order to establish a robust, reliable and feasible method (or panel of methods) for future zoo HAI and HAR studies. Equally important is the human dimension of the HAI (i.e. the response and/or perception of the HAI by the human involved), necessitating the utilisation of proven, reliable methods from the social sciences. However, the measurement of the human perspective, or the implications of the HAI or HAR for animal welfare or human well-being are beyond the scope of this review. Similarly, the important but under-investigated field of multi-zoo 
comparisons of husbandry factors involved in determining HARs warrants further investigation, and will likely benefit from the application of standardised methodology to assess HARs.

\subsection{The evaluation process}

Methods currently used to assess HAIs and HARs were determined through literature searches on Google Scholar, Proquest and Web of Science, prior to November 2017. The search criteria included "human animal", "keeper animal" or "caretaker animal" AND "interaction", "relationship" or "bond". Subsequently, "zoo", “captive", "companion", “domestic", "farm”, "agriculture", "laboratory" and "wild" were added to each combination. Relevant studies from the dataset generated by the search engines were then identified from their key words, paper titles, and abstract contents. Criteria for inclusion also required that papers specifically measured HAI or HAR, rather than it being a subsequent or potential finding of a larger study. Additionally, only original research articles were included; review papers were excluded. Since the purpose of this study was to determine and evaluate the scientific methods used to assess HAI and HARs in zoos from the perspective of the animals, only reports that presented empirically-determined data and analyses of HAI or HAR studies were included. Data pertaining to the assessment of HAIs or HARs using social science methods, or investigated welfare outcomes of an HAI or HAR without measuring the HAI itself, were excluded in order to focus on animal-directed measures of HAI and HAR. Unpublished research, theoretical discussions, or manuscripts written in a language other than English were excluded. There were no further searches following the initial search.

The HAI or HAR methods used in the studies were categorised by the animal environment (zoo, companion, agricultural, laboratory) and additionally categorised into the most commonly used methods, as shown in Table 1. Factors used to evaluate the zoo HAI or HAR methods (Table 2) were adapted from Waiblinger et al (2006) and could be of use within the zoo industry. The important aspect here is that the majority of the studies included in Tables 1 and 2 use the assessment of HAI to determine the HAR rather than measuring HAR empirically and so it is difficult to explain how these methods differ when measuring HAI or HAR. Employing the new definition of HAR, we are now able to measure 
HAR distinctly from HAI and have devised a scoring system to evaluate methods, based on our evaluation criteria (Table 3).

\subsection{Response-based tests}

\subsection{Units of measurement}

Latency (time taken) to respond and distance parameters are an ex situ mechanism to determine how animals respond to each other, or in the case of HAIs to measure their response to a human (Keeling 1995; McBride 1963; Stricklin 1979; Waiblinger et al 2006). For application to HAR assessment, latency and distance parameters could be used to compare responses by an animal towards different people, as well as being used in longitudinal studies to evaluate the nature of a HAR. Human-animal interaction tests such as 'avoidance distance' and 'voluntary approach' tests use latencies to measure an animal's reaction to a human, by recording the time taken for an animal to approach or avoid a human (Waiblinger et al 2006). However, latency to specific responses such as performing a requested behaviour (Ward and Melfi, 2015), latency to feed (Boissy and Bouissou, 1988), or latency to move to desired area (Breuer et al., 2003) can also be used as measures. Measuring distance parameters, most commonly the proximity to a human is accomplished through using a laser distance meter, or distance estimations (Martin and Melfi 2016, Sherwen et al 2014, Smith 2014). Animals which initiate or accept close contact with conspecifics may cluster in groups, whereas aversion (or displacement) from conspecifics will be exhibited as greater spacing of animals (Keeling 1995). These spatial patterns can indicate the choices an animal has made, taking fixed environmental barriers to proximity into account. It follows that proximity to humans, and an animal's decision to approach or move away from a human, will provide an indication of the animal's perception of the HAI, or HAR. Though, due to the knowledge that individual keeper-animal dyads can be established (Ward and Melfi, 2015), in order to truly understand the HAR, comparisons between different humans must be incorporated into methods using latency and distance parameters. Therefore, highlighting the need for a method that can incorporate this aspect with a degree of standardisation for use of these measures within the zoo environment. 
228 Latencies can be used during routine HAIs (e.g. husbandry tasks) to investigate the nature of the HAI, this involves measuring the time an animal takes to respond to a specific cue from a keeper which can be monitored and recorded from areas within close proximity, i.e. as if the researcher were a zoo visitor. Ward and Melfi (2015) described how a shorter latency was indicative of an animal's enhanced cooperation and representative of a positive HAI. However, it is possible that the animal could be responding out of fear; this emphasises the importance of measuring other parameters in order to differentiate affective states, or potential motivation for behaviours. Depending on the cue provided, recording the latency to respond requires no formal training routine, especially where behaviours have been linked to current husbandry practices (Ward and Melfi, 2013). Latency tests can also easily fit into an animal management routine and be recorded from a distance, therefore being safe for the observer and reducing the potential observer effect on the animal. However, the motivation with which an animal responds may affect its latency, and this may be influenced by either the HAR or unrelated factors (e.g. appetite, presence of conspecifics). Longitudinal testing, appropriate replication of tests, and/or comparisons between human-animal dyads is therefore necessary to determine HAR using these methods. In addition, the manner in which the observer first appears to the animal (e.g. suddenly, or from a specific direction; or the way that the human is dressed) may have an impact on latency, which may be difficult to quantify without repeated testing and longitudinal study designs.

Spatial parameters and latency to respond to a cue have also been used to assess HAIs and HARs between zookeepers and zoo-housed Chapmans zebra (Equus quagga chapmani), Sulawesi macaque (Macaca nigra) and black rhinoceros (Diceros bicornis) (Carlstead 2009; Ward \& Melfi 2013; 2015). A significant difference in animals' latency to respond appropriately (i.e. perform the required/requested behaviour) to cues and signals from different zookeepers was interpreted to indicate that unique zookeeper-animal dyads had been formed (Carlstead 2009; Ward \& Melfi 2013, 2015). In addition to measuring latency, Ward and Melfi $(2013 ; 2015)$ also measured the keepers' escalation (positive or negative advances) of the original cue to try and quantify any differences between the keepers, as well as performance from the animals. However, it could also be that the animal's latency 
varies according to the time of day, the mood of the animal on the day, or some form of environmental impact; therefore these potential factors would require either standardisation or at least measurement. Whilst this method has been utilised within the zoo context with a variety of species and within a multizoo set up (Ward \& Melfi 2013; 2015), it may be difficult to compare across species and zoos. This challenge arises because of differing animal management routines, different cues provided by keepers and different enclosure designs; each distinct request would likely elicit different periods in latencies and responses.

The influence of animal sociality must also be considered when using latencies to assess HAIs and HARs. For example, Ward and Melfi (2013) showed that socially housed animals (Chapmans zebra and Sulawesi black crested macaques) responded to keeper cues and commands by performing the requested behaviours significantly quicker than solitary animals (black rhinoceros). This could be dependent on the individual or a result of social facilitation, i.e. once one individual has performed the required behaviour others follow (Zentall 2006). It was predicted that the animal most likely to initiate a response within a group will demonstrate personality traits associated with confidence and boldness (Ward \& Melfi 2013). Battini et al (2016) investigated the validity and feasibility of multiple HAR tests in dairy goats. During HAI approach tests with dairy goats within a social housing system, the male bucks were generally the first to approach, and inhibited the approach behaviour of the female goats (Battini et al 2016). Whilst this social influence could compromise the HAI tests by reducing validity and feasibility, this still requires testing in a zoo setting, and highlights a potentially novel aspect of HAIs and HARs to elucidate in non-domestic species. In this instance, when investigating HAIs it would be beneficial to perform qualitative behaviour assessments (see below, section 3.5) and personality profiling (Wemelsfelder \& Lawrence 2001; Carlstead et al 1999b) for each individual in order to investigate the potential effect of hierarchy or personality on HAI and HAR. However, the impact that social facilitation and hierarchy are likely to have within a group renders the investigation of specific keeperanimal dyads (i.e. HAR) difficult to accomplish in socially housed zoo species. Moreover, separating individual animals for research purposes is also unlikely to be feasible, or ethical. 

and unfamiliar keeper ("Keeper for the Day"), to discover whether animals were able to distinguish between the two, and the influence on the animal's behavioural response. During routine HAI events, such as feeding and cleaning, observations of measurable animal behaviours were recorded including interactions and avoidance behaviours. In addition, estimations of the proximity of the animal to keeper were recorded $(<1 \mathrm{~m}, 1 \mathrm{~m},>1 \mathrm{~m})$ using scan sampling. This method allowed differences between responses with familiar and unfamiliar keepers to be detected, through a decrease in avoidance behaviour towards familiar keepers. Authors did not distinguish between the routine HAIs. For example, cleaning and feeding may have quite different effects on the animal with cleaning potentially perceived as negative/neutral, being associated with increased noise, smells of disinfectant and removal of their bedding, and feeding perceived as positive/neutral. In addition, other variables such as the clothing worn, and the presence of the unfamiliar keeper in combination with the familiar keeper need to be standardised in future studies to ensure that they are not influencing the results. There were significantly more positive HAIs (reported as 'physical contact') between animals and unfamiliar keepers than familiar keepers. This method was able to identify HAIs that could then be linked to the development of possible HARs for unfamiliar keepers. However, interpretation of findings with this method could be challenging due to the multiple potential mechanisms involved, such as curiosity towards an unfamiliar keeper, which could therefore influence the interpretation of the HAI and consequently the HAR. Additionally, it could be that a HAR between the familiar keepers and animals has already been established and therefore less need to reinforce the interaction, again suggesting that HARs could be objectively quantified using this method.

When using distance-based measures of a HAI, the method of estimating distance, or the use of broad distance categories varies between studies (e.g. Battini et al 2016; Dalla Costa et al 2015). This is likely to introduce inconsistencies and lower validity and accuracy between and even within studies if different observers are used to estimate distance, i.e. depending on the manner in which distance is estimated, or the size of the distance categories used. In order to generate accurate data for analysis, recording the positional parameter will require using appropriate apparatus to measure the distance 
between the animal and human during different events. These measurements could potentially be marked out onto the enclosure floor prior to behavioural observations. However, in turn there is potential that a changed, novel addition to an area may influence animal behavioural responses. In addition, prior marking may not always be practical depending on enclosure design, substrate, accessibility and other variables. Some zoo HAI studies have successfully used remote distance measuring devices (e.g. Sherwen et al 2014; 2015), which use a laser to record distance from the meter to a solid surface. However, the handling required of distance meters can potentially increase the risk of observers missing subtle cues or movements from the animals, compared to studies without this technology. Moreover, using a distance meter may not be feasible depending on zoo enclosure design and accessibility. Again, strict implementation of methods is needed to enable a full comparison of the animals' distance and therefore analysis of HAI.

Measuring the response to cues through latency and distance parameters can provide information on multiple features of HAIs that could contribute to the understanding of HARs within a zoo environment. Through the use of distance meters and conducting behavioural observations alongside latencies during routine HAI events this will increase validity and accuracy, and the practical application of this method. However, this method could prove difficult for multi-zoo comparisons due to different behavioural requests and enclosure accessibility between institutions.

The voluntary approach test was developed for horses by Søndergaard \& Halekoh (2003), and has been referred to as the "reaction to a stationary human" test (Waiblinger et al 2006). In this regard, it differs from the avoidance test in which the human approaches or attempts to touch an animal. An approach behaviour is defined as the animal approaching a stationary human; this can also be interpreted as the level of fear of humans an animal may have (Hemsworth \& Coleman 1998; de Passille \& Rushen 2005). There are variations in terms of the experimental procedure and variables measured in order to utilise this method, however the basic concept is the same. A test person enters an area and stands stationary, 
the latency of an animal's approach can then be recorded, or when observing a group of animals, the percentage of animals observed to approach the human within a fixed time is recorded. Consequently, the level of fear of humans can be interpreted from the variables measured and used to establish the nature of the HAI, or HAR if assessed longitudinally.

Battini et al (2016) found that measuring latency during a voluntary approach test (defined as the time interval between the stimulus and response in this case) was the most feasible indicator to measure quality of the HAI in dairy goats, when evaluated against avoidance distance test and sneezing, i.e. the number of alert sounds. However, the definition of an approach varies between studies; it may be defined as the first contact (goats: Battini et al 2016), the animal moving within a specific distance radius (dairy cows: Rousing \& Waiblinger 2004) or the first display of a species-specific approach behaviour (piglets; front leg and head in zone where person is sitting (De Oliveira et al 2015). It may be that different definitions and implementation strategies are required for different species, however, this makes it increasingly challenging to evaluate the most appropriate way of utilising and replicating this particular method in a standardized manner. Battini et al (2016) also used distance parameters to record the percentage of dairy goats that entered within a $1.5 \mathrm{~m}$ radius around the test person at 1 minute intervals, subsequent to the test person entering and standing stationary. The test person created a $1.5 \mathrm{~m}$ radius outline on the floor of the test area in order to easily record the number of individuals. However, the authors state that the feasibility of recording the distance parameter was reduced due to the time required to measure and mark out the semi- circumference on the test floor (Battini et al 2016). Other methods of demarcating the zone of interest may therefore be more appropriate. Nonetheless, the reaction to a stationary human is easily performed and frequently used for on-farm assessment (Waiblinger et al 2006). However, curiosity of a novel event such as a human's presence may increase the motivation to approach (Merchant et al 1997).

A study of the response of 12 ungulate species to a stationary human keeper, under two conditions (inside the enclosure and then outside the enclosure) was conducted by Thompson (1989). Behaviours categorised as either interactive or non-interactive were observed and recorded; the recipient (either 
another animal or human) of an interactive behaviour, visual orientation, and physical contact towards

367

the recipient were also scored. Behavioural observations were made outside of normal feeding times, with a keeper who was not the animals' normal keeper, and all had access to food ad libitum, with the aim of avoiding the potential confounder of keeper-food provisioning association existing for the animals (Thompson 1989).

Within this study there were instances of aggressive behaviour from some animals which resulted in the procedure and position of the stationary keeper needing to be altered to include a physical barrier and the cessation of data collection in some cases. This highlights the risks to both animal and human safety which will require consideration prior to using this test procedure to assess HAI and HARs in zoos. To ensure safety for both the keepers and animals involved, preliminary behavioural observations can be made, as well as the provision of a physical barrier. Smith (2014) included the use of approach behaviours as one of several prosocial human-directed behaviours by great apes towards both visitors and keepers in a zoo environment with a physical barrier. These affiliative behaviours were collectively classified as "close" $(<3 \mathrm{~m})$ or "distant" $(>3 \mathrm{~m})$, with positive interactions expected to be characterised by high levels of close affiliative behaviours. The degree of familiarity and close affiliative behaviours, including approach, were much greater in orangutans compared with gorillas, which may suggest this method was sensitive enough to detect a difference between species. However, other zoo environmental factors such as enclosure design and quality, group size and the availability of conspecifics within a group could also be influential, and therefore need to be considered.

Similarly, using quantitative measures in avoidance and approach tests to investigate an emotional state such as fear may be inappropriate. These tests are likely to elicit different behavioural reactions, which can be misinterpreted. For example, Zebu cattle fear responses can range from intense avoidance, active defence, or inhibition of movement ("freezing") (Burrow \& Corbet 2000). As such, the freezing behaviour of Zebu cattle may be misinterpreted using quantitative measures of distance to a human (i.e. as an animal having a good temperament and/or low fear). However, using qualitative and speciesspecific methods would better enable the identification of the fear response in this species (Burrow \& 
Corbet 2000) and would likely be of benefit to a wide range of zoo-housed species (see Qualitative

Behavioural Assessment section below).

396

Some agricultural HAI studies using the voluntary approach test involve the movement of the animal into a test area in order to minimise confounding variables and to ensure safety. However, this in itself could elicit a behavioural response from the animal prior to the test (De Oliveira et al 2015; Waiblinger et al 2006; Søndergaard \& Halekoh, 2003). In order to avoid strong fear reactions to a human entering the test area, the test procedure should include a period of habituation. (Battini et al, 2016). This method requires minimal financial cost and training, however safety inside the enclosure is the primary concern with this method. The practical application of the voluntary approach test would be highly dependent on the accessibility to animals, enclosure design, training and the time taken to perform the test.

The avoidance test was developed in an agricultural context, initially for cows (Waiblinger et al 2003), and has since been used and validated for a few species (dairy goats: Battini et al 2016; horses and donkeys: Dalla Costa et al 2015). This test involves a person approaching an animal, with an attempt to touch or handle the animal. The latency of the animal to avoid (e.g. walk away from) the human is recorded in addition to behavioural responses of the animal. The test ends when the animal withdraws and moves away from the human. The avoidance distance from a human can be defined as the minimum distance to which an animal will allow a moving human to approach. This is thought to reflect the previous experience of the animal, under the assumption that animals which are most fearful will maintain a greater distance (de Passille \& Rushen 2005). However, it could be possible that the manner in which the animal retreats could indicate more about the HAI than just the distance, however this has not been evaluated. In order to assess HARs using this method, the animal response would need to be compared using different humans, and will most likely necessitate multiple repeat testing to confirm findings. 
In zoos, avoidance behaviours have been investigated in terms of response to conspecifics, obstacles, and visitors (e.g gorillas: Collins and Marples, 2016, penguins: Sherwen et al 2015, polar bears: Renner \& Kelly 2006). However, these studies did not include empirical testing regarding the animal response to a specific HAI. There are currently no examples of using the avoidance test method in a zoo setting. This may be due to the ethical implications of creating a situation predicted to potentially elicit a fear response, or the safety risks involved with some species. Nonetheless, this method has been successfully used in monitoring HAIs between humans and horses whilst utilising a physical barrier to ensure safety (Dalla Costa et al 2015), and could therefore be used for species that are housed in a protected contact management system (i.e. management of animals from behind barriers). Dalla Costa et al (2015) estimated the distance between a horses' head and assessors hand in 'arm lengths'. Within a zoo setting, when considering HAIs with potentially dangerous species, the human may not be permitted to get within an arm's length of the animal, meaning if the animal doesn't move away from the physical barrier and the human does not approach further for safety reasons, the precise avoidance distance could not be assessed.

Battini et al (2016) assessed the feasibility of the avoidance method to determine its suitability for use as a farm welfare monitoring tool for dairy goats. Notably, the method was found to be time consuming on a large farm scale; it also required specific training by the observer to properly move into an area, recognise a first avoidance reaction, and assess the correct distance (Battini et al 2016). The interpretation of animal response can also be difficult if the animal did not move and neither approached nor avoided the human (Battini et al 2016, Rousing \& Waiblinger 2004). In light of the limitations identified for this method, it may not be feasible within a zoo setting due to the lack of standardisation.

Experimental procedures have been developed for certain species to allow the observation and evaluation of an animal's reaction to handling (Waiblinger et al 2006). Within domestic animal studies, these methods usually involve responses to leading or moving, capture, restraint, and specific handling 
events such as veterinary procedures (e.g. dairy cows: Waiblinger et al 2004; horses: Jezierski et al 1999; piglets: Brajon et al 2015; lambs: Caroprese et al 2012, e.g. poultry: Korte et al 1999). Both behavioural and physiological parameters can be measured during handling tests, such as time taken for a handling or restraint procedure, vocalisations, heart rate and circulating cortisol concentrations (e.g. in cattle, Waiblinger et al 2004; Lensick et al 2001). These tests rely on the animal being suitable for handling by humans in a safe manner (for both animal and humans). However, the requirement to include animal handling in the assessment also has ethical implications when conducted for research purposes, and opportunistic sampling may be limited for many zoo species due to the rarity of handling events.

In contrast, handling events including leading, moving, or capture are generally common practice in livestock husbandry, albeit with varying styles, frequency or intensity among farms. Given the variation in the degree of handling that animals will experience in zoos, typically depending on the species and the safety implications of human contact with them, the reaction to handling test may be not suitable in all zoo species. However, particular species are regularly handled during educational programmes within some zoological institutions; in these instances, investigating the nature of HAI would be a beneficial addition to welfare assessments of these animals (Baird et al 2016). Through measuring the response of animals that are subjected to routine handling and therefore repeated interactions, information about the HAR can be determined.

The lack of standardised handling procedures for the reaction to handling test due to varying species and management can result in additional influencing factors affecting and animal's response to humans therefore reducing reliability. The reaction to handling test has been used to directly assess the HAR through measuring response to humans, behavioural and physiological variables, and following different previous HAI treatments. Lensink et al (2001) measured heart rate, number of pushes from human, time to load the animal and number of buck-kicks during transportation loading in calves which had previously been subjected to either minimal human contact or daily human contact. Heart rate was a sensitive parameter which showed differences between calves which received additional previous 
human contact and calves subjected to minimal contact, however it must be noted that heart rate could have also been influenced by human presence and degree of locomotion. Measuring heart rate in zoo species could be accomplished through on-animal monitors or stethoscope measurements, however this is likely to prove challenging and not possible for some zoo species due to safety and ethical concerns. The study also found that housing systems influenced how calves reacted to humans during handling suggesting that other factors can also influence an animal's response to humans during a handling event.

In some zoos, animals undergo positive reinforcement training whereby the animal receives a reward in order to increase the frequency of a desired behaviour (Heidenreich 2007). Zoo professionals are then able to cue the animal to participate in medical or husbandry procedures. Assessing responses during training, including handling, may not be a true representation or measurement of the 'reaction to handling' but more the reaction to the training and/or a learned responses. Positive reinforcement training however, does increases the opportunity for positive HAIs and is therefore likely to increase positive HAR (Ward \& Melfi 2013) but would not be suitable as a method to measure the HAI or HAR.

The robustness and practical application of the response to handling test is species dependant, meaning this test cannot be used for all species within a zoological institution due to the safety implications of contact with certain animals. It also requires some standardisation in regards to how the animal is handled; the variation between handling style and skills of the handlers could potentially affect the results of the test (de Passille \& Rushen 2005), thereby decreasing reliability. Likewise, if this test is used with animals that are not handled as part of their daily routine, this method has the potential for negative ethical/welfare implications. Lastly, the additional time required of zoo staff to participate in this method would deem it unsuitable for long-term monitoring. Therefore, the response to handling test is considered unsuitable for use within zoo settings as a measure of HAIs or HARs. 
501 Qualitative Behaviour Assessment (QBA) is a "whole-animal" assessment of an animal, based on 502 evaluating body language and posture; it is used effectively to determine the animals' affective state, 503 their personality, temperament and individual behaviour profiles (Wemelsfelder \& Lawrence 2001, 504 Wemelsfelder 2000, 2001). However, it may be possible to adapt this method to assess HAIs and HARs 505 with specific familiar or unfamiliar humans. Qualitative behaviour assessments involve using freechoice profiling in which observers are asked to generate their own descriptive vocabularies of how an animal behaves, based on observing the whole animal's body language from numerous video clips (known as phase one). Subsequently, using these adjectives observers score the animal from these and additional video clips (phase two). However, due to the requirement for multiple observers to analyse video clips during the two phases of free-choice profiling, the practical application of the QBA method can be challenging, time consuming, and even costly (e.g. observer expenses, IT equipment). Alternatively, a validated fixed-list of terms can be determined and used during phase two. Clarke et al (2016) directly compared the use of a fixed list and free-choice profiling using the same videos of grouphoused sows and concluded that there was little difference. For application to HAR research, video clips which depict HAIs between animals and humans in a variety of settings or situations can be scored through free-choice profiling or using a fixed-list of descriptors. However, the fixed-list would remove the process of qualitatively interpreting the animals' expressions (Wemelsfelder 2009; Napolitano et al 2012), therefore free choice profiling would be preferred when measuring HAIs and HARs in the zoo environment, and would represent a novel application of this method. Whilst it may be possible to conduct QBA with live observations of animals (Wemelsfelder and Lawrence, 2001) in order to reduce recording logistics, ensuring that the number of observers required (around 20) does not impact on the

522 behaviour and/or response of the animals and keepers involved will be difficult (if not impossible).

523 Previous published studies utilising QBA have all used video footage and is also likely to be the most efficient form of observation in a zoo setting. 
525 Qualitative behaviour assessments have been applied to agricultural species as a cost-effective and 526 reliable approach to monitoring animal welfare (dairy cattle: Wemelsfelder et al 2009; horse and ponies;

527 Napolitano et al 2008; Wemelsfelder and Lawrence 2001; McMillan 2000; Morton 2000). The method 528 includes the incorporation of subtle movements, posture and aspects of the context in which the 529 behaviour occurs into an animal's overall style of behaviour; thereby evaluating the "animal-as-a530 whole" (e.g. bold, shy, hostile) (Napolitano et al 2008; Wemelsfelder et al 2001; 2000). There are few 531 HAI studies which use QBA, although this approach has been used to determine the nature of HAIs in 532 regard to stockperson handling style on dairy calves, and has also demonstrated the ability of 533 stockpersons to predict animal behaviour (Ellingsen et al 2014, Ebinghaus 2017). These assessments 534 have also been used to determine individual traits in zoo species (e.g. snow leopards; Gartner and Powel 2011), such as scores on "friendly to keeper", which can then be correlated with other factors such as breeding success and welfare (e.g. black rhinos: Carlstead et al 1999a; 1999b). Applying QBA as an HAI or HAR assessment method within zoos will elicit a more sensitive, integrative, "whole-body" assessment of how an animal interacts with humans in their environment, incorporating responses which may not be captured during quantitative assessments. This method comes the closest to being able to measure a HAR from the animal's perspective so long as it incorporates long term monitoring and the video footage enables observers to monitor the pattern of the interactions. Daily interactions, such as routine tasks for a particular species that occur with multiple keepers and animals can be observed using QBA to investigate HARs in terms of how the animal responds holistically to these repeated interactions. For example, the authors have observed footage during a daily husbandry routine whereby a giraffe was provided food by a keeper, the keeper attempted to touch the giraffe, the giraffe then pulled away and removed itself from the interaction and the food. Through using QBA, and therefore capturing the "whole body" response and affective state of the animal, this scenario could be more comprehensively documented and evaluated in accordance with our new definition including the content, quality and pattern of interactions. 
551 Some studies have combined the use of QBA and quantitative methods, such as behaviour frequencies

552 (e.g. Napolitano et al 2012; Rutherford et al 2012). This suggests QBA could be used alongside 553 quantitative data obtained from a HAI test.. By combining QBA with ethogram-derived data, it may be 554 possible to gain a better understanding of an animal's affective state during particular HAI events or assess the existence or character of a HAR. This is advantageous when assessing the nature of HARs at an individual level, which consequently may aid in understanding the potential impact personality, social facilitation and hierarchy have on HARs.

The practical application and feasibility of QBA is challenging. Recording the initial videos, especially if this requires specific HAI events to be observed, and the requirement to capture varying aspects of an animal's behavioural repertoire are key logistical factors to consider when implementing QBA. Nonetheless, logistical challenges can be overcome. Importantly, this method can be performed without contact or interference with the animal, and videos can typically be easily obtained for all species within a zoo environment, dependant on enclosure design and accessibility. High agreement among observer groups with varying backgrounds has been demonstrated in agricultural studies, and among keepers in the limited zoo studies that exist, proving QBA to be a reliable method to investigate HAIs as well as HARs.

Within the zoo environment where routine HAI events occur daily among multiple keepers and animals, QBA will enable subtle movements, posture and aspects of the context in which the behaviour occurs (which may otherwise be overlooked in quantitative methods) to be incorporated into HAI evaluations. Although means to validate QBA exist, and have been used in the few QBA zoo studies published to date, further testing is required to determine the validity and reliability of QBA for use in studies investigating the presence or characteristics of HAI and HARs. Therefore, applying QBA could elicit a

573 better understanding and interpretation of how HAIs can determine and influence HARs and warrants further investigation. 
577 Throughout this evaluation, common constraints and limitations have become apparent when 578 considering the application if these methods to a zoo environment with a wide variety of species and accessibility. External factors such as housing, social groupings, husbandry and environmental aspects may influence the results of the described tests. For example, varying responses to HAIs were elicited at different times of the year for lactating cows, which could be associated with altered husbandry practices, namely the variation in quality and quantity of HAIs during indoor and outdoor housing periods (Battini et al 2011). Seasonal husbandry practices, breeding or group dynamics have the potential to influence differences in avoidance distance; these factors may also be difficult to control within the zoo environment (Battini et al 2011; Waiblinger et al 2006; Thompson 1989), but would be worthwhile investigating.

Latency and distance parameters used in response to cues, voluntary approach and avoidance tests all measure the assumption that how an animal responds through performing a behaviour or moving represents how that animal perceives human presence or interaction. From this, the features of the HAI can be consequently used to determine the HAR. However, animal responses could also be the response to a different interaction or movement. It will also be difficult to know specifically whether the animal is responding to the human or coincidentally moving towards or facing a given direction for an unrelated reason. Curiosity of a novel event could also increase the motivation to approach or perform a requested behaviour in the presence of a human (Merchant et al 1997). In a zoo setting, this could suggest that this test is less suitable for animals that rarely have human contact, as it may be measuring animal curiosity rather than a HAI or an indicator of fear or personality (Chelluri et al 2013; Waiblinger et al 2003; Marchant et al 1997). Smith (2014) discussed the findings that apes tended to seek proximity to certain staff members such as waste disposal and education staff, even though the staff behaviours were not necessarily rewarding to the ape compared to zoo professionals that may feed them, for example. This suggested that an approach behaviour might indicate an interest or curiosity instead of familiarity or the anticipation of a reward. In a zoo setting, this could suggest that the voluntary approach and 
curiosity rather than a HAI or an indicator of fear (Chelluri et al 2013; Waiblinger et al 2003; Marchant et al 1997).

605

The safety risks for participants will prohibit the use of the voluntary approach, avoidance and reaction to handling tests for some zoo-housed species. Although there are studies within zoo settings in which the response of animals is observed in the presence of relatively stationary humans, such as zoo visitors, these situations are far from ideal since visitors are not stationary for consistent times, or may be part of a group with mixed activity, and are most often separated from the animal by some form of barrier (Sherwen et al 2014; Sherwen et al 2015; Chamove et al 1988). The voluntary approach and avoidance test have been used with the presence of a physical barrier to ensure safety with some agricultural and zoo species (ungulates; Thompson 1989, horses; Dalla Costa et al 2015). However, this still may not be feasible for some zoo species that are potentially dangerous and are managed via protected contact, therefore suggesting these tests may not be applicable to all zoo species.

In agricultural HAI and HAR research, specific tests have been extensively investigated in terms of reliability, validity, feasibility and effectiveness. However, although the current research on HAI and HARs in zoos is a growing area of scientific interest, methods of assessing these in zoos have not yet been standardised. Previous studies have inferred the HAR from the animal's perspective based on measuring HAIs alone. An extension of measuring HAI to HAR is not automatic, therefore emphasising the need for standard methods to measure HAI and HAR specifically across species, rather than erroneously using the terms interchangeably. Of the methods available and evaluated, three have been identified as having potential for successful application to measuring HAI in zoos. Following our evaluation criteria, measuring latency to respond (e.g. cue or command), QBA and the voluntary approach test are methods that are considered reliable and feasible tests to assess HAIs within a zoo environment. Perhaps more importantly, under the new HAR definition, these methods are considered likely to be of particular value when empirically measuring HARs. With some modifications, such as 
the use of physical barriers, these tests do not induce fear in the animals or risk the safety of the staff or animals involved. Due to the variation in species, husbandry practices and enclosures within the zoo environment, we recommend that each of the three methods identified here should be subjected to further testing in a zoo environment using the evaluation scoring factors adopted in this review. Our identification of three potential methods enables the progression of the study of HARs within a zoo environment, ultimately ensuring that the implications of HARs for animal welfare can be reliably investigated and compared.

\subsection{Animal welfare implications}

In zoos, research has identified that positive HAIs can lead to positive HARs, however no previous studies have empirically measured HARs due to the difficulties associated with the previous definition. The influence that these diverse HAIs have on an animal's welfare state has only recently started to be quantified. As such, practical and evidence-based recommendations are not available to ensure high animal welfare during HAIs. Data exist to demonstrate the overwhelming potential for HAI and HARs to exert significant impact on zoo animal welfare status, however these are derived primarily from preliminary pilot studies (in zoos) or extrapolation from more comprehensively conducted animal welfare studies in agricultural settings. Along with the new definition of HARs, this evaluation, based on a subjective assessment using defined criteria has highlighted three potential methods (qualitative behaviour assessment, latency to respond, and voluntary approach tests) that could be used to assess HARs within a zoo environment in order to empirically determine the impact that these may have on animal welfare.

\section{Acknowledgments}

The authors would like to thank Nottingham Trent University's QR funding for providing the financial support for this project. The authors would like to also thank Hon. Prof. Ellen Dierenfeld for her help proof-reading the manuscript for submission and the anonymous reviewers involved in the publication for stimulating additional discussions which led to the development of the new HAR definition. 
Baird BA, Kuhar CW, Lukas KE, Amendolaginea LA, Fuller GA, Nemet J, Willis MA, Schooka, MW 2016 Program animal welfare: Using behavioral and physiological measures to assess the well-being of animals used for education programs in zoos. Applied Animal Welfare Science 176 pp. 150-162

662

Baker KC 2004 Benefits of positive human interaction for socially-housed chimpanzees. Animal Welfare 13(2) pp. 239

665

Barongi R, Fisken FA, Parker M and Gusset M 2015 Committing to conservation: the world zoo and aquarium conservation strategy. Gland, Switzerland: WAZA Executive Office

668

Battini M, Andreoli E, Barbieri S. and Mattiello, S 2011 Long-term stability of Avoidance Distance tests for on-farm assessment of dairy cow relationship to humans in alpine traditional husbandry systems. Applied Animal Behaviour Science 135(4) pp. 267-270

Battini M, Barbieri S, Waiblinger S and Mattiello S 2016 Validity and feasibility of Human-Animal Relationship tests for on-farm welfare assessment in dairy goats. Applied Animal Behaviour Science 178 pp. $32-39$

676

Boissy A, Bouissou MF 1988 Effects of early handling on heifers' subsequent reactivity to humans and 679

Boivin X, Le Neidre P, Chupin, JM 1992 Establishment of cattle-human relationship. Applied Animal Behaviour Science Amsterdam 32(4) pp. 325- 335

682

Brajon S, Laforest JP, Bergeron R, Tallet C and Devillers N 2015 The perception of humans by piglets: recognition of familiar handlers and generalisation to unfamiliar humans. Animal Cognition 18(6) pp. 
687 Breuer K, Hemsworth PH, Coleman GJ 2003 The effect of positive or negative handling on the 688 behavioural and physiological responses of nonlactating heifers. Applied Animal Behaviour Science. 84 pp. $3-22$

690

Burrow HM and Corbet NJ 2000 Genetic and environmental factors affecting temperament of zebu and zebu-derived beef cattle grazed at pasture in the tropics. Australian Journal of Agricultural Research. 55 pp. $155-162$

694

Carlstead K, Mellen J \& Kleiman DG 1999a Black rhinoceros (Diceros bicornis) in U.S. zoos: I. 696 Individual behavior profiles and their relationship to breeding success. Zoo Biology 18 pp. 17-34

697

Carlstead K, Fraser J, Bennett C, Kleiman DG 1999b Black rhinoceros (Diceros bicornis) in U.S. Zoos, II. Behavior, breeding success, and mortality in relation to housing facilities. Zoo Biology 18 (1) pp. $35-52$

701

Carlstead K 2009 A Comparative Approach to the study of Keeper-Animal Relationships in the Zoo. Zoo Biology 28 pp. 589-608

704

Carlstead K, Paris S, Brown J L 2018 Good keeper-elephant relationships in North American zoos are mutually beneficial to welfare. Applied Animal Behaviour Science in press.

Caroprese M, Napolitano F, Boivin X, Albenzio M, Annicchiarico G and Sevi A 2012 Development

Carrasco L, Colell M, Calvo M, Abello MT, Velasco M and Posada S 2009 Benefits of training/playing therapy in a group of captive lowland gorillas (Gorilla gorilla gorilla). Animal Welfare 18 pp9-19 
Chamove AS, Hosey GR, and Schaetzel P 1988 Visitors excite primates in zoos. Zoo Biology 7, pp. $359-369$

716

Chelluri GI, Ross SR and Wagner KE 2013 Behavioral correlates and welfare implications of informal interactions between caretakers and zoo-housed chimpanzees and gorillas. Applied Animal behaviour Science 147 (3-4) pp 306-315

Clarke T, Pluske J R, and Fleming P A 2016 Are observer ratings influenced by prescription? A comparison of Free Choice Profiling and Fixed List methods of Qualitative Behavioural Assessment. Applied Animal Behaviour Science 177 pp. 77-83

725

Collins CK, and Marples NM 2016 The effects of zoo visitors on a group of Western lowland gorillas

(Gorilla gorilla gorilla) before and after the birth of an infant at Dublin Zoo. International Zoo Yearbook 50(1) pp.183-192

Dalla Costa E, Dai F, Murray LAM, Guazzetti S, Canali E and Minero M 2015 A study on validity and reliability of on-farm tests to measure human-animal relationship in horses and donkeys. Applied Animal Behaviour Science 163 pp. 110-121 63

de Passillé AM and Rushen J 2005 Can we measure human-animal interactions in on-farm animal 
Ebinghaus A, Ivemeyer S, Lauks V, Santos L, Brugemann K 2017 How to measure dairy cows’ responsiveness towards humans in breeding and welfare assessments? A comparison of selected behavioural measures and existing breeding traits. Applied Animal Behaviour Science 196 pp. $22-29$

Ellingsen K, Coleman GJ, Lund V, Mejdell C.M. 2014 Using qualitative behaviour assessment to explore the link between stockperson behaviour and dairy calf behaviour. Applied Animal Behaviour Science 153 pp. $10-17$

Ellingsen K, Zanella AJ, Bjerkås E and Indrebø A, 2010 The relationship between empathy, perception of pain and attitudes toward pets among Norwegian dog owners. Anthrozoös 23(3) pp.231-243

751

Estep DQ and Hetts S 1992 Interactions, relationships and bonds: the conceptual basis for scientistanimal relations. In: H. Davis \& A. D. Balfour (Eds.), The inevitable bond: examining scientist-animal interactions (pp. 6-26). CAB International. Cambridge, UK.

Fravel L 2003 Critics Question Zoos' Commitment to Conservation. National Geographic News.

[online] Accessed from: http://news.nationalgeographic.com/news/2003/11/1113 031113 zoorole.html.

Gartner MC and Powel D 2011 Personality assessment in Snow Leopards (Uncia uncia). Zoo Biology 29 pp. $1-15$

Heidenreich B 2007 An Introduction to Positive Reinforcement Training and Its Benefits. Journal of Exotic Pet Care Medicine 16 (1) pp. 19-23

Hemsworth PH and GJ Coleman 1998 Human-Livestock Interactions. CAB, Wallingford, UK 
Hemsworth PH, Barnett JL and Coleman GJ 1993 The human-animal relationship in agriculture and its consequences for the animal. Animal Welfare 2(1) pp. 33-51

Hinde RA 1976 Interactions, relationships and social structure. Man pp. 1-17

Hosey G 2008 A preliminary model of human-animal relationships in the zoo. Applied Animal Behaviour Science 109(2) pp. 105-127

Hosey G, Melfi V 2014 Human-Animal Interactions, Relationships and Bonds: A Review and Analysis of the Literature. International Journal of Comparative Psychology 27 (1) pp. 117-142

Jezierski T, Jaworski Z, Gorecka A 1999 Effects of handling on behaviour and heart rate in Konik horses: Comparison of stable and forest reared youngstock. Applied Animal Behaviour Science 62 (1) pp. $1-11$

Jones RB 1996 Fear and adaptability in poultry: insights, implications and imperatives. World's Poultry Science Journal 52 (2) pp. 131-174

Jones RB 1997 Fear and distress. In: M. C. Appleby, B. O. Hughes (Eds), Animal Welfare, CAB International, Wallingford pp. 75-87

Keeling LJ 1995 Spacing behaviour and an ethological approach to assessing optimal space allocations for laying hens. Applied Animal Behaviour Science 44 pp. 171-186

Kennedy JS 1992 The New Anthropomorphism. Cambridge University Press, Cambridge

Korte SM, Ruesink W, Blokhuis HJ 1999 Heart rate variability during manual restraint in chicks from high and low feather pecking lines of laying hens. Physiol. Behav. 65 pp. 649-652 
797 Lensink BJ, Raussi S, Boivin X. Pyykkönen M and Veissier I 2001 Reactions of calves to handling depend on housing condition and previous experience with humans. Applied Animal Behaviour Science 70(3) pp. 187-199

800

Marchant JN, Burfoot A, Corning S and Broom DM 1997 The 'human approach test' - a test of 802 fearfulness or investigatory behaviour. In: Proceedings of the 31st International Congress of the International Society for Applied Ethology, Prague, Czech Republic pp. 182

804

Marinelli L, Adamelli S, Normando S and Bono G 2007 Quality of life of the pet dog: Influence of owner and dog's characteristics. Applied Animal Behaviour Science 108(1) pp.143-156

807

Martin RA and Melfi V 2016 A Comparison of Zoo Animal Behavior in the Presence of Familiar and

Unfamiliar People. Journal of Applied Animal Welfare Science pp.1-11

810

Mcbride G, James JW and Soffner RN 1963 Social forces determining spacing and head orientation in a flock of domestic hens. Nature 197 pp. 1272-1273

McMillan FD 2000 Quality of life in animals. Journal of American Veterinary Medicine Association.

Meagher RK 2009 Validity and values as a tool for animal welfare research. Applied Animal Behaviour Science 119 pp. 1

Meehan CL, Mench JA, Carlstead K, Hogan JN 2016 Determining Connections between the Daily 
Mellen JD, 1991 Factors influencing reproductive success in small captive exotic felids (Felis spp.): a multiple regression analysis. Zoo biology 10(2) pp.95-110

825

Morton DB 2000 A systematic approach for establishing humane endpoints. Ilar Journal 41(2) pp. 80-

86

Napolitano F, De Rosa G, Grasso F and Wemelsfelder F 2012 Qualitative behaviour assessment of dairy buffaloes (Bubalus bubalis). Applied Animal Behaviour Science 141(3) pp. 91-100

831

Napolitano F, De Rosa G, Braghieri A, Grasso F, Bordi A, and Wemelsfelder F 2008 The qualitative assessment of responsiveness to environmental challenges in horses and ponies. Applied Animal

O'Haire M 2010 Companion animals and human health: Benefits, challenges, and the road ahead.

Pizzutto CS, Nichi M, Correa SR, Ades C. and Guimaraes MABV 2007 Reduction of abnormal primate newsletter $46(3)$ pp.6-9

Podberscek A, Blackshaw J, and Beattie A 1991 The effects of repeated handling by familiar and unfamiliar people on rabbits in individual cages and group pens. Applied Animal Behaviour Science 28 pp. $365-373$

846

847 Renner MJ and Kelly AL 2006 Behavioral decisions for managing social distance and aggression in captive polar bears (Ursus maritimus). Journal of Applied Animal Welfare Science 9(3) pp. 233-239 
Rousing T and Waiblinger S 2004 Evaluation of on-farm methods for testing the human-animal relationship in dairy herds with cubicle loose housing systems - - test-retest and inter-observer reliability and consistency to familiarity of test person. Applied Animal Behaviour Science 85 pp. 215-231

853

Rutherford KMD, Donald RD, Lawrence AB, Wemelsfelder F 2012 Qualitative Behavioural

Sherwen SL, Magrath MJL, Butler KL, Phillips CJC and Hemsworth PH 2014 A multi-enclosure study investigating the behavioural response of meerkats to zoo visitors. Applied Animal Behaviour Science 156 pp. $70-77$

860

Sherwen SL, Magrath MJL, Butler KL and Hemsworth PH 2015 Little penguins, Eudyptula minor, show increased avoidance, aggression and vigilance in response to zoo visitors. Applied Animal Behaviour Science 168 pp. 71-76

864

Smith JJ 2014 Human-animal relationships in zoo-housed orangutans (P. abelii) and gorillas (G.g. gorilla): The effects of familiarity. American Journal of Primatology 76 (10) pp: 942-955

867

Søndergaard E and Halekoh U 2003 Young horses' reactions to humans in relation to handling and social environment. Applied Animal Behaviour Science 84 pp. 265-280

Species360 (2016) [online] zims.Species360.org. Accessed on 22/11/2016

Stricklin WR, Graves HB and Wilson L 1979 Some theoretical and observed relationships of fixed and portable spacing behaviours of animals. Applied Animal Ethology 5 pp. 201-214 
Tetley C and O'Hara S 2012 Ratings of animal personality as a tool for improving the breeding, management and welfare of zoo animals. Animal Welfare 21(4) pp. 463-476

Thompson VD 1989 Behavioural response of 12 ungulate species in captivity to the presence of humans. Zoo Biology 8 pp. 275-297

Touma C and Palme R 2005 Measuring Fecal Glucorticoid Metabolites in Mammals and Birds: The Importance of Validation. Annuals New York Academy of Sciences 1046 pp. 54-74

Vitztum C and Urbanik J 2016 Assessing the dog: A theoretical analysis of the companion animal's actions in human-animal interactions. Society and Animals 24(2) pp. 172-185

Waiblinger S, Menke C and Fölsch DW 2003 Influences on the avoidance and approach behaviour of dairy cows towards humans on 35 farms. Applied Animal Behaviour Science 84(1) pp. 23-39

Waiblinger S, Menke C Korff J and Bucher A 2004 Previous handling and gentle interactions affect behaviour and heart rate of dairy cows during a veterinary procedure. Applied Animal Behaviour Science 85(1) pp.31-42

Waiblinger S, Boivin X, Pederson V, Tosi M, Janczak AM, Visser EK, and Jones RB 2006 Assessing the human-animal relationship in farmed species: A critical review. Applied Animal Behaviour Science 101 pp. $185-242$

Walsh F 2009 Human-animal bonds I: The relational significance of companion animals. Family process $48(4)$ pp. $462-480$

Ward SJ and Melfi V 2013 The implications of husbandry training on zoo animal response rates. Applied Animal Behaviour Science 147(1) pp. 179-185 
906 Ward SJ and Melfi V 2015 Keeper-Animal Interactions: Differences between the Behaviour of Zoo

907 Animals Affect Stockmanship. PloS one 10(10)

908

909

Watters JV and Powell DM 2012 Measuring animal personality for use in population management in zoos: suggested methods and rationale. Zoo Biology 31 pp. 1-12

911

912

Watters JV and Wielebnowski N 2009 Introduction to the special issue on zoo animal welfare. Zoo Biology 28(6) pp. 501-506

914

915

Wemelsfelder F, Hunter EA, Mendl MT, Lawrence AB 2000 The spontaneous qualitative assessment of behavioural expressions in pigs: first explorations of a novel methodology for integrative animal welfare measurement. Applied Animal Behaviour Science. 67 pp. 193-215

Wemelsfelder F and Lawrence AB 2001 Qualitative assessment of animal behaviour as an on-farm welfare-monitoring tool. Acta Agriculturae Scandinavica Section. A. 51 pp. 21-22

921

922

Wemelsfelder F, Hunter TEA, Mendl MT, and Lawrence AB 2001 Assessing the 'whole animal': a free choice profiling approach. Animal Behaviour 62 pp. 209-220

Wemelsfelder F, Millard F, Rosa G and Napolitano F 2009 Qualitative Behaviour Assessment. In: B. Forkman, L. Keeling (Eds.), Assessment of Animal Welfare Measures for Dairy Cattle, Beef Bulls and Veal Calves, Cardiff University, UK pp. 21

Wemelsfelder F, Hunter A E, Paul E S, Lawrence A B 2012 Assessing pig body language: agreement and consistency between pig farmers, veterinarians and animal activists. Journal of Animal Science 90 pp. 3652-3665 
933 Whitham JC and Weilebnowski N 2009 Animal-bases welfare monitoring: using keeper ratings as an 934 assessment tool. Zoo Biology 28 pp. 454-560

935

936 Wielebnowski NC 1999 Behavioral differences as predictors of breeding status in captive cheetahs. Zoo Biology 18(4) pp. 335-349

938

Wielebnowski NC, Fletchall N, Carlstead K, Busso JM, and Brown JL 2002 Noninasive assessment of adrenal activity associated with husbandry and behavioural factors in the north american clouded leopard population. Zoo Biology 21 pp. 77-98

942

943

Windschnurer I, Boivin, Waiblinger S 2009 Reliability of an avoidance distance test for the assessment of animals responsiveness to humans and a preliminary investigation of its association with farmers attitudes on bull fattening farms. Applied Animal Behaviour Science 117 pp. 117-127

Zentall TR 2006 Imitation: Definitions, evidence, and mechanisms. Animal Cognition 9 pp. 335-353

Table Captions:

951 Table 1. Current studies and methods to assess human-animal-relationships in various animal groups

Table 2. Factors used for evaluation of methods to measure human-animal-relationships in zoos 